\title{
The Search for an Alternative Biomarker for the Detection and Diagnosis of Hepatocellular Carcinoma - The Journey
}

\section{So Far}

\author{
Hussaini IM*, Musa B, Bello A \\ Department of Microbiology, Faculty of Life Sciences, Ahmadu, Bello University, \\ Zaria, Nigeria
}

*Corresponding author: Hussaini Ibrahim Mohammed, Department of

\section{Review Article \\ Volume 1 Issue 3}

Received Date: November 04, 2017

Published Date: December 06, 2017

Microbiology, Faculty of Life Sciences, Ahmadu Bello University, Zaria, Nigeria, Tel: +2348142446864; Email: hussainiibrahim269@gmail.com

\section{Abstract}

Hepatocellular carcinoma (HCC) is one of the most common cancers in African where hepatitis is endemic. HCC has become the third most common malignancy worldwide, with a very poor prognosis, rendering it the fourth highest cause of cancer - related deaths. The poor prognosis of HCC is mostly linked to late diagnosis because few treatment strategies can be implemented in patients with advanced disease. The levels of AFP combined with abdominal ultrasonography were considered the "gold standard" for HCC screening in patients with liver cirrhosis. Several studies have suggested other biomarkers that are more sensitive, specific and reliable compared to AFP and USS in HCC diagnosis. These biomarkers include Proteins (including enzymes and isoenzymes), growth factors and their receptors, molecular markers and cytokines. These biomarkers could be useful biomarkers or complementary biomarkers in the diagnosis of liver cirrhosis associated hepatocellular carcinoma.

Keywords: Hepatocellular Carcinoma; Biomarker; Alpha Fetoprotein; Hepatitis; Ultrasonography

\section{Introduction}

Hepatocellular carcinoma (HCC) is one of the most frequently diagnosed cancers worldwide and the most common malignant tumors in the world. The incidence of HCC is steadily increasing worldwide however the disease predominantly occurs in Asia and Africa [1].

Oncogene addiction is important in the proliferation and survival of cancer cells. Oncogene addiction is the activation of specific oncogenes and inactivation of specific tumor suppressors, such as $\mathrm{Rb} 1$ in retinoblastoma [2] and BRCA1 in breast cancer. So far, specific oncogene addictions are yet to be observed in HCC, which is a complex disease with a variety of underlying pathogenic anomalies caused by multiple risk factors. The lack of ideal biomarkers for HCC diagnosis, prognosis, and therapy has posed a major challenge to HCC management [3].

HCC is the fifth most common malignant tumor and the third leading cause of cancer-related deaths. Worldwide, there are about 626,000 new HCC cases and nearly 600,000 HCC-related deaths each year with an incidence equal to the death rate $[4,5]$.

Advances in tumor biology have brought about increased interest in identifying molecular biomarkers of HCC. New cutting-edge technologies such as nextgeneration sequencing and microarray technologies 
have led to the search for biomarkers into a new era of "omics". With the aid of such technologies, it is now quite easy to examine a whole tumor genome, transcript to me, proteome, epigenome, metabolome, and miRNA profile and analysis of tens of thousands of molecular targets is now affordable and operable. Currently, numerous circulating biomarkers and tissue biomarkers have been identified; however, few biomarkers are acceptable for clinical utility because of their low predictive accuracy and/or high cost [3].

Biomarkers are molecular indicators of biological status detectable in body fluid such as blood and urine or tissue. They are useful for the clinical management of various disease states. Biomarkers could also serve as a measurement tool to detect disease presence and progression and to guide more targeted therapy. This is achievable by monitoring the threshold concentrations of biomarkers [6].

Serum tumor biomarkers have been used as an effective and reliable method of detection of malignant tumors in addition to ultrasonography and computer tomography because of convenience, cost effectiveness and accuracy of these techniques. The effectiveness of screening techniques for HCC can be improved by using the appropriate single or combination of tumor markers [8]. The current standard surveillance for HCC includes a combination of 6-monthly ultrasound scans (USS) and serum $\alpha$-fetoprotein (AFP) measurement; the latter is considered the 'gold standard' for HCC detection [7].

Alpha-fetoprotein (AFP) a glycoprotein produced by the fetal liver and yolk sac during pregnancy was the first serologic assay used in the detection and clinical follow up of patients with hepatocellular carcinoma. Although the serum levels of AFP are often elevated in HCC, it is not always the case. AFP levels may be elevated initially in the early stages of HCC and then drop or even normalize before rising again as disease progression occurs. Elevated serum AFP levels has also been recognized in the presence of acute and chronic viral hepatitis as well as in patients with cirrhosis caused by hepatitis $\mathrm{C}$. In general, consistently elevated serum AFP levels greater than $500 \mathrm{ng} / \mathrm{mL}$ are indicative of HCC. Lower serum concentrations which are only transient in nature are more often present in benign liver disease. Furthermore, in some cases of HCC, AFP elevations are not apparent at all [6].AFP can be analyzed using an Elecsys 2010 auto-analyzer system or by Axsym using micro particle enzyme immunoassay (MEIA) technology.

\section{Why the search for an alternative biomarker for the detection and diagnosis of hepatocellular carcinoma}

HCC in patients with history of chronic hepatitis or cirrhosis characterized by continuous inflammation and regeneration of hepatocytes. The coexistence of inflammation and cirrhosis in HCC makes its early diagnosis and prognostic assessment much more difficult. Hence the need to identify valuable biomarkers for the diagnosis and treatment of HCC [8].

Curative therapeutic practices for HCC currently are surgical resection, liver transplantation and local ablation. Analysis of serum alpha-fetoprotein (AFP) levels combined with imaging techniques such as magnetic resonance imaging, ultrasonography and computerized tomography are used for the diagnosis of HCC without pathologic confirmation. However, improvement is needed in times of early diagnosis since only $44 \%$ of HCC patients are diagnosed at a localized disease stage and moreover only about $30 \%$ of patients with HCC are candidates for potentially curative treatments at the time of diagnosis. As such discovery of an effective, reliable tool for early diagnosis of HCC to increase the number of patients who are suitable for curative treatment will play a pivotal role in improving HCC patients' prognosis [3,9].

AFP has been regarded as the most useful serum protein thus far for patients at risk for HCC. However, its sensitivity for detecting HCC ranges between $25 \%$ $60 \%$, and its specificity is also low because serum AFP can also be detected in patients with cirrhosis $(11 \%$ $47 \%)$ and chronic hepatitis (15\%-58\%) [3,8,10,11].

AFP may also be elevated in benign chronic liver diseases, e.g. chronic viral hepatitis and liver cirrhosis without HCC, it was suggested that the 'gold standard' approach lacked sensitivity and specificity [12].

Serum AFP levels of more than $400 \mathrm{ng} / \mathrm{mL}$ which is considered diagnostic are observed only in a small percentage of patients with HCC. Moreover ultrasound surveillance even when performed at every three monthly intervals cannot improve detection of small HCC because of limitations in recall procedures $[13,14]$.

\section{Requirements of a biomarker for early diagnosis HCC}

A good biomarker for early diagnosis of HCC should fulfill the following requirements [3]: 
High accuracy: A biomarker meant for early diagnosis of HCC should achieve high accuracy. This would increase the probability of a diagnosis being made prior to spread and thus increase the cure rate.

Easily operable and non-invasive specimen: Collection of specimen for biomarker detection should be easily operable and non-invasive.

Cost-effectiveness: the cost-effectiveness of the biomarker should be put in to consideration.

Biomarkers from body fluids such as serum, plasma, urine, and bile are suitable candidates for early diagnosis of HCC because they are easily accessible. However Tumor tissue-oriented markers are not highly practical because not all tumor tissues can be obtained at an early stage and the invasive procedure may cause spread of tumor cells [15].

\section{Alternative biomarker for the detection and diagnosis of hepatocellular carcinoma}

The emergence of new cutting edge technologies has led to the search and discovery of biomarkers for HCC diagnosis. Several studies have suggested other biomarkers that are more sensitive, specific and reliable compared to AFP and USS in HCC diagnosis. These biomarkers include proteins (including enzymes and isoenzymes), growth factors and their receptors, molecular markers and cytokines.

\section{Proteins}

In addition to AFP, several serum proteins have been reported to have clinical significance in early diagnosis of HCC and are proved to have advantages over AFP.

Dickkopf-1 (DKK1): It is a member of secreted proteins family that play important role in HCC progression by promoting cytoplasmic/nuclear accumulation of beta-catenin in HCC cells via the Wnt/beta-catenin signaling pathway [16]. Serum DKK1 is a promising candidate for HCC diagnosis because it has better diagnostic value for HCC than does AFP, especially for patients with AFP-negative and early stage HCC [17]. However DKK1 is not overly specific for HCC diagnosis and a recent study reported that serum DKK1 was also elevated in patients with intrahepatic cholangiocarcinoma [18].This protein can be detected by indirect peptide ELISA for DKK-1 auto antibodies.

Golgi protein 73 (GP73): GP73 is a transmembrane glycoprotein with a molecular weight of $73 \mathrm{kDa}$ hence the name. It normally resides within the Golgi complex and is expressed in normal biliary epithelial cells however normal hepatocytes do not express this protein. So also the expression of this protein is significantly increased in liver diseases such as HCC [19].

Serum GP73 is a valuable biomarker for patients with HCC The GP73 level significantly increased in patients with HCC compared with healthy controls, decreased following surgical resection of HCC lesions and increased with tumor recurrence [20,21]. The sensitivity and specificity of serum GP73 for HCC were $74.6 \%$ and $97.4 \%$, respectively, compared with $58.2 \%$ and $85.3 \%$ for AFP [21]. GP73 is also known Golgi Phosphoprotein (GOLPH2).This protein can be detected by ELISA.

Protein induced by vitamin $\mathrm{K}$ absence or antagonist II (PIVKA-II): PIVKA-II is an abnormal prothrombin that has been widely proposed to be a useful HCC biomarker. As a screening test for detecting HCC, PIVKA-II yielded sensitivity and specificity values $(52.8 \%$ and $98.8 \%$, respectively) that were comparable with AFP [3]. It also known as Des-Gamma-Carboxy (Abnormal) Prothrombin (DCP). This protein can be detected by ELISA.

\section{Glycoprotein Antigens}

Glypican-3 (GPC3): GPC3is a membrane-anchored heparin sulfate proteogly can that has been demonstrated to interact with growth factors and modulate their activities. GPC3 binds to the cell membrane through the glycosylphosphatidylinositol anchors. Significant up regulation of GPC3 mRNA was observed in tumor tissues of HCC compared to paraneoplastic liver tissue, liver tissues of healthy adults, and liver tissues of patients with nonmalignant hepatopathy. Studies have shown that the expression of GPC3was significantly higher in the serum of HCC patients compared to that in the serum of healthy adults or patients with nonmalignant disease. It can be detected in $40-53 \%$ of HCC patients and $33 \%$ of HCC patients seronegative for both AFP and Des-gammacarboxyprothrombin (DCP). Soluble GPC3 (sGPC3) i.e. the $\mathrm{NH}$-terminal portion of GPC3, was reported to be superior to AFP in the sensitivity of detecting well or moderately differentiated HCC, and the simultaneous determination of both markers improves overall sensitivity from $50 \%$ to $72 \%$ [22].This biomarker can be detected by ELISA.

\section{Enzymes and Isoenzymes}

Gamma-Glutamyl Transferase (GGT): GGT is an enzyme that is mainly secreted by hepatic Kupffer cell and endothelial cell of bile duct in healthy adults and its activity increases in HCC tissues. Sensitivities of GGTII (a type of GGT isoenzymes) have been reported to be $74.0 \%$ in detecting large HCC and $43.8 \%$ in detecting small HCC. Sensitivity can be significantly improved 
with the simultaneous determination of GGTII, DCP, and AFP [23]. Fluorescence, high-performance liquid chromatography (HPLC) and electrophoresis have also been successfully employed in the detection of the activity of GGT.

Serum Alpha-1-Fucosidase (AFU): AFU is a liposomal enzyme that hydrolyzes fucose glycosidic linkages of glycoprotein and glycolipids. It is found in all mammalian cells and its activity increases in the serum of HCC patients $(\mathrm{nmol} / \mathrm{mL} / \mathrm{h})$ compared with that in the serum of healthy adults $(\mathrm{nmol} / \mathrm{mL} / \mathrm{h}$,), patients with cirrhosis $(\mathrm{nmol} / \mathrm{mL} / \mathrm{h})$, and patients with chronic hepatitis $(\mathrm{nmol} / \mathrm{mL} / \mathrm{h})$. It has been reported that the sensitivity and specificity of AFU at the cut-off value of $870 \mathrm{nmol} / \mathrm{mL} / \mathrm{h}$ were $81.7 \%$ and $70.7 \%$, respectively [6]. 7600 Clinical Analyzer can be used to detect this biomarker.

Human Carbonyl Reductase 2: Human Carbonyl Reductase 2 is an enzyme expressed in the human liver and kidney. It is important in detoxification of the reactive alpha-dicarbonyl compounds and reactive oxygen species deriving from oxidative stress in HCC. The human carbonyl reductase 2 levels have been shown to be inversely correlated to the pathological grading of HCC [24]. ELISA kit can be used to detect this biomarker.

\section{Growth Factors and Their Receptors}

Transforming Growth Factor-Beta (TGF- $\beta$ ): TGF- $\beta$ belongs to a super family of polypeptide signaling molecules that are involved in regulating cell growth, differentiation, angiogenesis, invasion, and immune function, TGF- $\beta$ is a predominant form of growth factor family in humans. Its mRNA and protein are over expressed in HCC compared with surrounding liver tissues, especially in small and well-differentiated HCCs [25]. Serum TGF- $\beta$ level has been found to be elevated in HCC patients compared to healthy adults or patients with nonmalignant liver disease [8]. ELISA kit can be used to detect this biomarker.

Tumor-Specific Growth Factor (TSGF): TSGF is released by malignant tumors release into peripheral blood during their growing period. Serum levels of TSGF may reflect the existence of tumor. TSGF can be used as a diagnostic marker in detecting HCC, and its sensitivity can reach $82 \%$ at the cut-off value of $62 \mathrm{U} / \mathrm{mL}$ and may have a higher accuracy with the simultaneous determination of other tumor markers. The simultaneous determination of TSGF (at the cut-off value of $65 \mathrm{U} / \mathrm{mL}$ ), AFP (at the cut-off value of 25 $\mathrm{ng} / \mathrm{mL}$ ), and serum ferritin (at the cut-off value of 240 $\mathrm{ng} / \mathrm{mL}$ ) can reach a sensitivity and specificity of $98.4 \%$ and $99 \%$, respectively [8].
Other growth factors and their receptors reported to be important in HCC diagnosis include: Epidermal Growth Factor Receptor (EGFR), Hepatocyte growth factor/scatter factor (HGF/SF) and Basic Fibroblast Growth Factor [6]. ELISA kit can be used to detect this biomarker.

\section{Molecular Markers (Circulating Nucleic Acids: mRNAs)}

Changes in levels of DNA, RNA and nucleosomes have been associated with tumor burden and progression of malignancy [26]. Circulating nucleic acids have been extensively studied with regard to their significance in diagnosis [27-30].

The level of plasma AFP mRNA is considered a diagnostic marker for HCC. Accumulating evidence has shown that micro RNAs (miRNAs) play important roles in cancer initiation, propagation, and progression [3]. Deregulation of miRNA occurs at early stages of HCC and increases throughout the various steps of hepatocarcinogenesis [28]. The plasma miRNA panel has a considerable clinical value for the early diagnosis of HCC and could help patients who might have otherwise missed the curative treatment window benefit from optimal therapy [30].

Analysis of circulating nucleic acids in plasma offers another avenue for noninvasive monitoring of a variety of physiological and pathologic conditions [31]. Several applications based on the detection of circulating cellfree nucleic acids in human plasma have been reported for the management of malignancies. The fundamental principle underlying these applications relates to the detection of extracellular nucleic acid molecules derived from diseased organs in plasma. Analysis of cell-free plasma RNA offers an opportunity for the development of pathology related markers [32-34].

This cell-free nucleic acids include: AlphaFetoprotein mRNA Alpha-Fetoprotein mRNA (AFP mRNA) [6]; Gamma-Glutamyl Transferase mRNA (GGT mRNA) [8]; Insulin-Like Growth Factor II (IGF-II) mRNA [35]; Albumin mRNA [36]; Micro RNAs (miRNAs) [37].

However, the diagnostic value of miRNAs is limited by one or more of the following factors: limited number of screened miRNAs, small sample size, failure to differentiate HCC from hepatitis, and lack of independent validation [3].

Recently, [3] measured plasma miRNA expression profiles (723 miRNAs) in a large cohort of 934 participants that included healthy individuals and patients with chronic HBV infection, cirrhosis, or HBVrelated HCC. They identified a miRNA panel (miR-122, miR-192, miR- 21, miR-223, miR-26a, miR-27a, and 
miR-801) that provided high diagnostic accuracy for discriminating patients with HCC from the healthy population (AUC $=0.941$ ) and patients with chronic HBV (AUC $=0.842$ ) or cirrhosis $($ AUC $=0.884)$. Their finding led to the conclusion that the plasma miRNA panel had considerable clinical value for the early diagnosis of HCC and could help patients who might have otherwise missed the curative treatment window benefit from optimal therapy.

\section{Cytokines}

Cytokines play an important role in the regulation and propagation of inflammatory responses and the homeostasis of organ functions [38]. In the liver, cytokines co-ordinate physiologic and pathologic processes such as liver growth and regeneration, as well as inflammatory processes during viral liver disease, liver fibrosis, and cirrhosis. Cytokines modulate the immune system and exert direct antiviral activity. Specifically, interleukins (IL)-1, -2 and -6 , as well as tumor necrosis factor (TNF)-a are all released in increased amounts during acute fulminate viral hepatitis; in this instance, they exert both proinflammatory and cytotoxic effects [7] carried out a study which shows the need to integrate the screening of cytokine profiles (specifically of IL-6 and IL-17) and vitamin D levels into the classic AFP profiling during clinical assessments of patients with HCV and cirrhosis in order to increase the prognostic and diagnostic sensitivity and specificity and hopefully aid in the prevention of development of HCC.IL-6 and IL-17 levels can be determined by enzyme Immunoassay or Enzyme Linked Immunosorbent Assay (ELISA).

\section{References}

1. Jemal A, Bray F, Center MM, Ferlay J, Ward E, et al. (2011) Global cancer statistics. CA Cancer J Clin 61(2): 69-90.

2. Sachdeva UM and O'Brien JM (2012) Understanding $\mathrm{pRb}$ : toward the necessary development of targeted treatments for retinoblastoma. J Clin Invest 122(2): 425-434.

3. Zhu K, Dai Z, Zhou J (2013) Biomarkers for hepatocellular carcinoma: progression in early diagnosis, prognosis, and personalized therapy. Biomarker Research 1(1): 10.

4. Parkin DM, Bray F, Ferlay J, Pisani P (2001) Estimating the world cancer burden: Globocan 2000. Int J Cancer 94(2): 153-156.

5. Parkin DM, Bray F, Ferlay J and Pisani P (2005) Global cancer statistics, 2002. CA Cancer J Clin 55(2): 74-108.
6. Behne T, Copur MS (2012) Biomarkers for Hepatocellular Carcinoma. International Journal of Hepatology Pp: 1-7.

7. Hammad LN, Abdelraouf SM, Hassanein FS, Mohamed WA and Schaalan MF (2013) Circulating IL-6, IL-17 and vitamin D in hepatocellular carcinoma: Potential biomarkers for a more favorable prognosis?. Journal of Immunotoxicology 10(4): 380-386.

8. Zhou L, Liu J and Luo F (2006) Serum tumor markers for detection of hepatocellular carcinoma. World J Gastroenterol 12(8): 1175-1181.

9. Bruix J, Llovet JM (2002) Prognostic prediction and treatment strategy in hepatocellular carcinoma. Hepatology 35(3): 519-524.

10. Tangkijvanich $P$, Anukulkarnkusol N, Suwangool $P$, Lertmaharit S, Hanvivatvong O, et al. (2000) Clinical characteristics and prognosis of hepatocellular carcinoma: analysis based on serum alpha fetoprotein levels. J Clin Gastroenterol 31(4): 302308.

11. El-Serag HB, Marrero JA, Rudolph L, Reddy KR (2008) Diagnosis and treatment of hepatocellular carcinoma. Gastroenterology 134(6): 1752-1763.

12. Tai WC, Hu TH, Wang JH, Hung $\mathrm{CH}$, Lu SN, et al. (2009) Clinical implications of a-fetoprotein in chronic hepatitis C. J Formos Med Assoc 108(3): 210-218.

13. Farinati F, Marino D, De Giorgio M, Baldan A, Cantarini M, et al. (2006) "Diagnostic and prognostic role of alpha fetoprotein in hepatocellular carcinoma both or neither?. Am J Gastroenterol 101(3): 524-532.

14. Trinchet JC, Chaffaut C, Bourcier V, Degos F, Henrion J, et al. (2011) Ultrasonographic surveillance of hepatocellular carcinoma in cirrhosis: a randomized trial comparing 3-and 6 month periodicities. Hepatology 54(6): 1987-1997.

15. Singhal A, Jayaraman M, Dhanasekaran DN and Kohli V (2012) Molecular and serum markers in hepatocellular carcinoma: predictive tools for prognosis and recurrence. Crit Rev Oncol Hematol, 82(2): 116-140.

16. Yu B, Yang X, Xu Y, Yao G, Shu H, et al. (2009) Elevated expression of DKK1 is associated with cytoplasmic/nuclear beta-catenin accumulation and poor prognosis in hepatocellular carcinomas. J Hepatol 50(5): 948-957. 
17. Shen Q, Fan J, Yang XR, Tan Y, Zhao W, et al. (2012) Serum DKK1 as a protein biomarker for the diagnosis of hepatocellular carcinoma: a large-scale, multicentre study. Lancet Oncol13 (8): 817-826.

18. Shi RY, Yang XR, Shen QJ, Yang LX, Xu Y, et al. (2012) High expression of dickkopf-related protein 1 is related to lymphatic metastasis and indicates poor prognosis in intrahepatic cholangiocarcinoma patients after surgery. Cancer 119(5): 993-1003.

19. Kladney RD, Cui X, Bulla GA, Brunt EM and Fimmel CJ (2002) Expression of GP73, a resident Golgi membrane protein, in viral and nonviral liver disease. Hepatology 35(6): 1431-1440.

20. Riener MO, Stenner F, Liewen H, Soll C, Breitenstein S, et al. (2009) Golgi phosphoprotein 2 (GOLPH2) expression in liver tumors and its value as a serum marker in hepatocellular carcinomas. Hepatology 49(5): 1602-1609.

21. Mao Y, Yang H, Xu H, Lu X, Sang X, et al. (2010) Golgi protein 73 (GOLPH2) is a valuable serum marker for hepatocellular carcinoma. Gut 59(12): 16871693.

22. Shirakawa H, Suzuki H, Shimomura M, Kojima $M$, Gotohda N, et al. (2009) Glypican-3 expression is correlated with poor prognosis in hepatocellular carcinoma. Cancer Sci 100(8): 1403-1407.

23. Cui R, He J, Zhang F, Wang B, Ding H, et al. (2003) Diagnostic value of protein induced by vitamin $\mathrm{K}$ absence (PIVKAII) and hepatoma-specific band of serum gamma-glutamyl transferase (GGTII) as hepatocellular carcinoma markers complementary to $\alpha$-fetoprotein. Br J Cancer 88(12): 1878-1882.

24. Shan Liu, Lijie Ma, Weixue Huang, Yin Shai, Xiaona Ji, et al. (2006) Decreased expression of the human carbonyl reductase 2 gene HCR2 in hepatocellular carcinoma. Cellular and Molecular Biology Letters 11(22): 230-241.

25. Okumoto K, Hattori E, Tamura E, Shinichi Kiso, Hisayoshi W et al. (2004) Possible contribution of circulating transforming growth factor- $\beta 1$ to immunity and prognosis in unrespectable hepatocellular carcinoma. Liver International 24(1): 21-28.

26. Zhou J, Shi YH and Fan J (2012) Circulating cell-free nucleic acids: promising biomarkers of hepatocellular carcinoma. Semin Oncol 39(4): 440448.
27. Cho WC (2011) Circulating microRNAs as minimally invasive biomarkers for cancer theragnosis and prognosis. Front Genet 2: 7.

28. Gao P, Wong CC, Tung EK, Lee JM, Wong CM, et al. (2011) Deregulation of microRNA expression occurs early and accumulates in early stages of HBV-associated multistep hepatocarcinogenesis. J Hepatol 54(6): 1177-1184.

29. Llovet JM, Pena CE, Lathia CD, Shan M, Meinhardt G, et al. (2012) Plasma biomarkers as predictors of outcome in patients with advanced hepatocellular carcinoma. Clin Cancer Res 18(8): 2290-2300.

30. Zhou J, Yu L, Gao X, Hu J, Wang J, et al. (2011) Plasma microRNA panel to diagnose hepatitis $B$ virus-related hepatocellular carcinoma. J Clin Oncol 29(36): 4781-4788.

31. Poon RTP, Ng IO, Lau C, Yu WC, Fan ST, et al. (2001) Correlation of serum basic fibroblast growth factor levels with clinicopathologic features and postoperative recurrence in hepatocellular carcinoma. American Journal of Surgery 182(3): 298-304.

32. Lo YMD and Chiu RWK (2004) The biology and diagnostic applications of plasma RNA," Annals of the New York Academy of Sciences 1022: 135-139.

33. Chan AKC, Chiu RWK, Lo YMD, Clinical Sciences Reviews Committee of the Association of Clinical Biochemists (2003) Cell-free nucleic acids in plasma, serum and urine: a new tool in molecular diagnosis. Annals of Clinical Biochemistry 40(Pt 2): 122-130.

34. Anker $P$ and Stroun $M$ (2002) Progress in the knowledge of circulating nucleic acids: plasma RNA is particle-associated. Can it become a general detection marker for a cancer blood test? Clinical Chemistry 48(8): 1210-1211.

35. Tsai JF, Jeng JE, Chuang LY, You HL, Wang LY, et al. (2005) Serum insulin-like growth factor-II as a serologic marker of small hepatocellular carcinoma. Scandinavian Journal of Gastroenterology 40(1): 68-75.

36. Cheung ST, Fan ST, Lee YT, Chow JP, Ng IO, et al. (2008) Albumin mRNA in plasma predicts posttransplant recurrence of patients with hepatocellular carcinoma. Transplantation 85(1): 81-87. 


\section{Bioequivalence \& Bioavailability International Journal}

37. Ferracin M, Veronese A, Negrini M (2010) Micro markers: MiRNAs in cancer diagnosis and prognosis. Expert Rev Mol Diagn 10(3): 297-308.

38. Denning TL, Wang YC, Patel SR, Williams IR, Pulendran B (2007) Lamina propria macrophages and dendritic cells differentially induce regulatory andIL-17-producing T-cell responses. Nat Immunol 8(10): 1086-1094. 